\title{
Correlation Effects and the High-Frequency Spin Susceptibility of an Electron Liquid: Exact Limits
}

\author{
G. S. Atwal and N. W. Ashcroft \\ Cornell Center for Materials Research, and the Laboratory of Atomic and Solid State Physics, \\ Cornell University, Ithaca, New York 14853-2501
}

(Dated: November 16, 2018)

\begin{abstract}
Spin correlations in an interacting electron liquid are studied in the high-frequency limit and in both two and three dimensions. The third-moment sum rule is evaluated and used to derive exact limiting forms (at both long- and short-wavelengths) for the spin-antisymmetric local-field factor, $\lim _{\omega \rightarrow \infty} G_{-}(\mathbf{q}, \omega)$. In two dimensions $\lim _{\omega \rightarrow \infty} G_{-}(\mathbf{q}, \omega)$ is found to diverge as $1 / q$ at long wavelengths, and the spin-antisymmetric exchange-correlation kernel of time-dependent spin density functional theory diverges as $1 / q^{2}$ in both two and three dimensions. These signal a failure of the local-density approximation, one that can be redressed by alternative approaches.
\end{abstract}

PACS numbers:

\section{INTRODUCTION}

The detailed incorporation of exchange and correlation (xc) into the dynamic response of an interacting electron liquid remains a challenge to theory. The mean field approach of the random phase approximation (RPA), which neglects xc effects, becomes increasingly inadequate as both the dimensionality and density of electrons is lowered. Sum rules provide exact constraints on the response of an electron liquid and thus are extremely useful as tests of any scheme that purports to include xc effects. Alternatively they can be used to constrain particular approximations such as those embodied in local field factors $G_{ \pm}(\mathbf{q}, \omega)$. The static behavior of both the spinsymmetric (+) and spin-antisymmetric (-) local field factors has been well studied ${ }^{\underline{1}}$ and various fitting formulae have been proposed that conform to the known exact constraints 2.3.4. Their dynamic forms have attracted a great deal of recent attention since they exhibit far richer behavior than their static counterparts.

However, up until now, most dynamical studies have focussed on the spin-symmetric case; in this paper we report on a derivation of the exact behavior of the dynamic spin-antisymmetric response in the high- $\omega$ limit within the linear response scheme. In particular, the first and third-moment sum rules are derived (Sec III) and then used in Sec III to determine the exact asymptotic behaviour of $\lim _{\omega \rightarrow \infty} G_{-}(\mathbf{q}, \omega)$ in the low- and high- $q$ limit. The xc kernel in time-dependent density functional theory is closely related to these local field factors and in Sec IV it will be shown that the high-frequency spinantisymmetric xc kernel must then diverge in the long wavelength limit. This divergence demonstrates that the dynamical xc potential in time-dependent spin-density functional theory (TDSDFT), as applied to a homogeneous system, must be a nonlocal functional of the local density.

\section{MOMENT SUM RULES}

To describe the formalism needed to derive the moment sum rules of an electron liquid we will first need the electron particle-hole operator $\hat{\rho}_{\mathbf{k} \sigma}(\mathbf{q})$, defined as

$$
\hat{\rho}_{\mathbf{k} \sigma}(\mathbf{q})=c_{\mathbf{k} \sigma}^{\dagger} c_{\mathbf{k}+\mathbf{q}, \sigma},
$$

where $c_{\mathbf{q} \sigma}^{\dagger}$ and $c_{\mathbf{q} \sigma}$ are the fermionic creation and annihilation operators, respectively, and $\sigma$ denotes the spin projection. The standard one-particle density operators are then

$$
\hat{\rho}_{\sigma}(\mathbf{q})=\sum_{\mathbf{k}} \hat{\rho}_{\mathbf{k} \sigma}(\mathbf{q}), \quad \hat{\rho}(\mathbf{q})=\sum_{\mathbf{k} \sigma} \hat{\rho}_{\mathbf{k} \sigma}(\mathbf{q}),
$$

and, for the spin equivalent,

$$
\hat{s}(\mathbf{q})=\sum_{\mathbf{k} \sigma} \eta_{\sigma} \rho_{\mathbf{k} \sigma}(\mathbf{q}), \quad \eta_{\sigma}=\left\{\begin{array}{lll}
+1 & \text { for } & \sigma=\uparrow \\
-1 & \text { for } & \sigma=\downarrow
\end{array}\right.
$$

In the thermodynamic limit the Hamiltonian for a $d$ dimensional system of $N$ electrons immersed in a uniform neutralizing background of volume $\Omega$ is given by $(\hbar=1)$,

$$
H=\sum_{\mathbf{q} \sigma} \omega_{0}(\mathbf{q}) c_{\mathbf{q} \sigma}^{\dagger} c_{\mathbf{q} \sigma}+\frac{N}{2 \Omega} \sum_{\mathbf{q} \neq 0} V(\mathbf{q})\left[\frac{1}{N} \hat{\rho}(\mathbf{q}) \hat{\rho}(-\mathbf{q})-1\right]
$$

where $\omega_{0}(\mathbf{q})=q^{2} / 2 m$, and $V(\mathbf{q})$ is the Fourier transformed Coulomb potential, i.e. $V(\mathbf{q})=4 \pi e^{2} / q^{2}$ for $d=3$, and $V(\mathbf{q})=2 \pi e^{2} / q$ for $d=2$. From linear response theory the retarded charge-density $\chi_{C}$ and spindensity $\chi_{S}$ responses are given by

$$
\chi_{C}(\mathbf{q}, t)=\frac{i}{\Omega}\langle[\hat{\rho}(\mathbf{q}, t), \hat{\rho}(-\mathbf{q}, 0)]\rangle \theta(t),
$$

and

$$
\chi_{S}(\mathbf{q}, t)=\frac{i}{\Omega}\langle[\hat{s}(\mathbf{q}, t), \hat{s}(-\mathbf{q}, 0)]\rangle \theta(t),
$$

where $\theta(t)$ is the Heaviside step function and \langle\rangle denotes averaging over the equilibrium ensemble as specified by 
the Hamiltonian in Eq. (4). Note that to obtain a physical spin-spin response from $\chi_{S}(\mathbf{q}, t)$ in Eq. (6) a factor $g^{2} \mu_{B}^{2} / 4$ is required where $g$ is the gyromagnetic ratio and $\mu_{B}$ is the Bohr magneton.

As usual, the spectral representation of $\chi(\mathbf{q}, \omega)$, the Fourier transform of $\chi(\mathbf{q}, t)$, can be obtained from

$$
\chi(\mathbf{q}, \omega)=\int_{-\infty}^{\infty} \frac{d \omega^{\prime}}{\pi} \frac{\operatorname{Im} \chi\left(\mathbf{q}, \omega^{\prime}\right)}{\omega^{\prime}-\omega-i 0^{+}} .
$$

Analyticity of $\chi_{S}(\mathbf{q}, \omega)$ in the upper half of the complex$\omega$ plane requires that $\operatorname{Im} \chi_{S}(\mathbf{q}, \omega)$ be an odd function of $\omega$ and so, in the the high- $\omega$ limit, $\chi_{S}(\mathbf{q}, \omega)$ expands as

$$
\lim _{\omega \rightarrow \infty} \chi_{S}(\mathbf{q}, \omega)=-\sum_{l=1}^{\infty} \frac{\left\langle\omega^{(2 l-1)}\right\rangle}{\omega^{2 l}}
$$

where the moments $\left\langle\omega^{(l)}\right\rangle$ are given by

$$
\begin{aligned}
\left\langle\omega^{(l)}\right\rangle & \equiv \int_{-\infty}^{\infty} \frac{d \omega}{\pi} \omega^{l} \operatorname{Im} \chi_{S}(\mathbf{q}, \omega) \\
& =\frac{1}{\Omega}\left\langle\left[\left(i \frac{\partial}{\partial t}\right)^{l} \hat{s}(\mathbf{q}, t), \hat{s}(-\mathbf{q}, 0)\right]\right\rangle_{t=0}
\end{aligned}
$$

The first moment yields the well known $f$-sum rule,

$$
\left\langle\omega^{(1)}\right\rangle=2 n \omega_{0}(\mathbf{q})
$$

where $n \equiv N / \Omega$ is the macroscopic homogeneous particle density. The derivation of the $f$-sum rule invokes number conservation via the continuity equation,

$$
\frac{\partial \rho_{\sigma}(\mathbf{q}, t)}{\partial t}=-i \mathbf{q} \cdot \mathbf{j}_{\sigma}(\mathbf{q}, t)
$$

where $\mathbf{j}_{\sigma}(\mathbf{q}, t)$ is the current density fluctuation operator for particles with spin $\sigma$

$$
\mathbf{j}_{\sigma}(\mathbf{q}, t)=\frac{1}{m} \sum_{\mathbf{k}}\left(\mathbf{k}+\frac{1}{2} \mathbf{q}\right) \rho_{\mathbf{k} \sigma}(\mathbf{q}, t)
$$

Thus number density is separately conserved for electrons with spin up and down since there is no term in the equilibrium Hamiltonian Eq. (4) which can flip spin. That correlation effects play no role in the first moment may be understood by noting that in the high- $\omega$ limit electrons which interact via a velocity-independent potential cannot be influenced by the effects of others when the time interval is infinitesimal.

The third-moment sum rule $e^{5}$, after straightforward but lengthy calculations, gives 6.7

$$
\left\langle\omega^{(3)}\right\rangle=2 n \omega_{0}(\mathbf{q})\left[\omega_{0}^{2}(\mathbf{q})+\omega_{0}(\mathbf{q})(12 / d)\left\langle E_{K E}\right\rangle+I_{d}(\mathbf{q})\right]
$$

where $\left\langle E_{K E}\right\rangle$ is the average kinetic energy per electron in the interacting system and $I_{d}(\mathbf{q})$ is given by

$$
I_{d}(\mathbf{q})=\frac{1}{m} \sum_{\mathbf{k} \neq 0} V(\mathbf{k})(\hat{\mathbf{q}} \cdot \mathbf{k})^{2}[\tilde{S}(\mathbf{q}-\mathbf{k})-S(\mathbf{k})],
$$

where for $\mathbf{q} \neq 0$

$$
\begin{aligned}
& \tilde{S}(\mathbf{q})=N^{-1}\langle\hat{s}(\mathbf{q}) \hat{s}(-\mathbf{q})\rangle \\
& S(\mathbf{q})=N^{-1}\langle\hat{\rho}(\mathbf{q}) \hat{\rho}(-\mathbf{q})\rangle
\end{aligned}
$$

define the static magnetic and usual structure factors, respectively. For completeness, we express the structure factors in terms of the spin-resolved pair correlation functions,

$$
\tilde{S}(k)-1=\frac{n}{2} \int d^{2} r\left(g_{\uparrow \uparrow}(r)-g_{\uparrow \downarrow}(r)\right) \exp (-i \mathbf{k} \cdot \mathbf{r}),
$$

and

$$
S(k)-1=\frac{n}{2} \int d^{2} r\left(g_{\uparrow \uparrow}(r)+g_{\uparrow \downarrow}(r)-2\right) \exp (-i \mathbf{k} \cdot \mathbf{r})
$$

where $g_{\uparrow \uparrow}(r)$ and $g_{\uparrow \downarrow}(r)$ are the spin-parallel and antiparallel contributions, respectively, to the electron pair correlation function $g(r)$, i.e.

$$
g(r)=\frac{1}{2}\left[g_{\uparrow \uparrow}(r)+g_{\uparrow \downarrow}(r)\right]
$$

It has been noted 8 that the third-moment sum rule differs for the charge and spin density response, in contrast to the first moment sum rule which is obeyed by both $\chi_{C}$ and $\chi_{S}$. The physical root of this qualitative difference can be inferred from the continuity equation for longitudinal spin-current, used in the derivation of Eq. (13), namely

$$
i \frac{\partial}{\partial t} \mathbf{q} \cdot \mathbf{j}_{\sigma}(\mathbf{q}, t)=\sum_{\mathbf{k}}\left[\omega_{0}(\mathbf{q}+\mathbf{k})-\omega_{0}(\mathbf{k})\right]^{2} \rho_{\mathbf{k} \sigma}(\mathbf{q}, t)+\frac{1}{\Omega} \sum_{\mathbf{k} \mathbf{p}} V(\mathbf{p}) \frac{\mathbf{q} \cdot \mathbf{p}}{m} c_{\mathbf{k} \sigma}^{\dagger}(t) \rho(\mathbf{p}, t) c_{\mathbf{k}-\mathbf{p}+\mathbf{q} \sigma}(t)
$$

Hence, it can be shown that the presence of the interelectron potential allows a transfer of momentum density across spin-up and spin-down electrons. Thus, although the total momentum density must be conserved, the mo- 
mentum density of each spin species is not ${ }^{9}$. This is why the third-moment sum rule, essentially an expression of momentum and number density conservation, must differ for charge and spin density susceptibilities.

An expression for $I_{d}(\mathbf{q})$ has been known for some time for three-dimensional systems ${ }^{7}$, and here we just record the limiting expressions,

$$
I_{3}(\mathbf{q} \rightarrow 0)=\frac{\omega_{\mathrm{p}}^{2}(\mathbf{q})}{3}\left[1-g_{\uparrow \downarrow}(0)\right],
$$

and

$$
I_{3}(\mathbf{q} \rightarrow \infty)=\frac{\omega_{\mathrm{p}}^{2}(\mathbf{q})}{3}\left[1-2 g_{\uparrow \downarrow}(0)\right]
$$

where $\omega_{\mathrm{p}}(\mathbf{q})=\left[2 n \omega_{0}(\mathbf{q}) V(\mathbf{q})\right]^{1 / 2}$ is the plasma frequency. Note that in Eqs. (21] 22) we have applied $8 g_{\uparrow \uparrow}(0)=0$, and that the high-q limit is to be taken such that $0 \ll$ $\omega(\mathbf{q}) \ll \omega$.

In two dimensions we find

$$
I_{2}(\mathbf{q})=\frac{e^{2}}{2 m} \int_{0}^{\infty} d k k\{R(\mathbf{q}, \mathbf{k})[\tilde{S}(k)-1]-k[S(k)-1]\}
$$

with

$$
R(\mathbf{q}, \mathbf{k})=\int_{0}^{2 \pi} \frac{d \theta}{\pi} \frac{q^{2}+k^{2} \cos ^{2} \theta+2 k q \cos \theta}{\left(q^{2}+k^{2}+2 k q \cos \theta\right)^{1 / 2}}
$$

The limiting behavior is then

$$
\begin{aligned}
I_{2}(\mathbf{q} \rightarrow 0) & =\frac{e^{2}}{2 m} \int_{0}^{\infty} d k k^{2}[\tilde{S}(k)-S(k)], \\
I_{2}(\mathbf{q} \rightarrow \infty) & =-\frac{1}{2} \omega_{\mathrm{p}}^{2}(\mathbf{q}) g_{\uparrow \downarrow}(0) .
\end{aligned}
$$

\section{LOCAL-FIELD FACTORS}

Another way to express the correlation effects in the spin response is to introduce the notion of a local-field factor, $G_{-}(\mathbf{q}, \omega)$, which modifies the effective field felt by a single electron, as expressed by

$$
\chi_{S}(\mathbf{q}, \omega)=-\frac{\bar{\chi}_{0}(\mathbf{q}, \omega)}{1+V(\mathbf{q}) G_{-}(\mathbf{q}, \omega) \bar{\chi}_{0}(\mathbf{q}, \omega)} .
$$

Here $\bar{\chi}_{0}(\mathbf{q}, \omega)$ is the modified Lindhard function for the interacting system, i.e. where exact interacting occupation numbers, $\bar{n}(\mathbf{k})$, are used, namely

$$
\bar{\chi}_{0}(\mathbf{q}, \omega)=\frac{1}{\Omega} \sum_{\mathbf{k}} \frac{\bar{n}(\mathbf{k})-\bar{n}(\mathbf{k}+\mathbf{q})}{\omega+\omega_{0}(\mathbf{k})-\omega_{0}(\mathbf{k}+\mathbf{q})+i 0^{+}} .
$$

The $\bar{n}(\mathbf{k})$ therefore replace the usual non-interacting fermion occupation number, $n(\mathbf{k})$, in the original Lindhard function, $\chi_{0}(\mathbf{q}, \omega)$. Since the exact occupation numbers are generally unknown we follow Ref. 10 and introduce a separate local factor, $G_{n}$ to write the modified Lindhard function in terms of the original Lindhard function $\chi_{0}$, i.e.

$$
\bar{\chi}_{0}(\mathbf{q}, \omega)=\frac{\chi_{0}(\mathbf{q}, \omega)}{1+V(\mathbf{q}) G_{n}(\mathbf{q}, \omega) \chi_{0}(\mathbf{q}, \omega)} .
$$

Thus we have

$$
\chi_{S}(\mathbf{q}, \omega)=-\frac{\chi_{0}(\mathbf{q}, \omega)}{1+V(\mathbf{q}) \bar{G}_{-}(\mathbf{q}, \omega) \chi_{0}(\mathbf{q}, \omega)}
$$

where $\bar{G}_{-}(\mathbf{q}, \omega)=G_{-}(\mathbf{q}, \omega)+G_{n}(\mathbf{q}, \omega)$.

The third-moment sum rule can again be utilised to determine the high- $\omega$ limit of the local-field factors, i.e. $\lim _{\omega \rightarrow \infty} G(\mathbf{q}, \omega)=G^{(\infty)}(\mathbf{q})$. First we will require the high- $\omega$ expansion of the Lindhard function, i.e.

$$
\lim _{\omega \rightarrow \infty} \chi_{0}(\mathbf{q}, \omega)=\frac{2 n \omega_{0}(\mathbf{q})}{\omega^{2}}+\frac{2 n \omega_{0}^{2}(\mathbf{q})}{\omega^{4}}\left[\omega_{0}(\mathbf{q})+\frac{12}{d}\left\langle E_{K E}\right\rangle_{0}\right]+O\left(\frac{1}{\omega^{6}}\right)
$$

where $\left\langle E_{K E}\right\rangle_{0}$ is the average kinetic energy per electron in a non-interacting system. After insertion of Eq. (31) into the high- $\omega$ expansion of the spin-density response Eq. (30) we observe that manifestations of the local field factor appear at $O\left(1 / \omega^{4}\right)$, i.e.

$$
\lim _{\omega \rightarrow \infty} \chi_{S}(\mathbf{q}, \omega)=-\frac{2 n \omega_{0}(\mathbf{q})}{\omega^{2}}-\frac{2 n \omega_{0}^{2}(\mathbf{q})}{\omega^{4}}\left[\omega_{0}(\mathbf{q})+\frac{12}{d}\left\langle E_{K E}\right\rangle_{0}-V(\mathbf{q}) 2 n \bar{G}_{-}^{(\infty)}(\mathbf{q})\right]+O\left(\frac{1}{\omega^{6}}\right) .
$$


of the local-field factor must be given by

$$
\bar{G}_{-}^{(\infty)}(\mathbf{q})=-\frac{I_{d}(\mathbf{q})}{\omega_{\mathrm{p}}^{2}(\mathbf{q})}+\frac{6}{\operatorname{dnV}(\mathbf{q})}\left(\left\langle E_{K E}\right\rangle_{0}-\left\langle E_{K E}\right\rangle\right)
$$

The kinetic part of the above expression gives a diverging contribution in the large $q$ limit which, previous authors 8 have argued, must be absorbed by a proper treatment of the self-energy of the proper polarizability function, i.e. the modified Lindhard function. The introduction of the additional local-field factor, $G_{n}$, allows us to demonstrate this explicitly, and indeed a high- $\omega$ expansion of Eq. (28) together with the definition of the local fieldfactor, Eq. (30), gives

$$
G_{n}^{(\infty)}(\mathbf{q})=\frac{6}{d n V(\mathbf{q})}\left(\left\langle E_{K E}\right\rangle_{0}-\left\langle E_{K E}\right\rangle\right),
$$

leaving the desired result,

$$
G_{-}^{(\infty)}(\mathbf{q})=-\frac{I_{d}(\mathbf{q})}{\omega_{\mathrm{p}}^{2}(\mathbf{q})}
$$

This result can also be derived from the work of Zhu and Overhauser ${ }^{11}$ based on the equation-of-motion $\operatorname{approach}^{12}$. In three dimensions the limiting forms are

$$
\begin{aligned}
G_{-}^{(\infty)}(\mathbf{q} \rightarrow 0) & =\frac{1}{3}[2 g(0)-1], \\
G_{-}^{(\infty)}(\mathbf{q} \rightarrow \infty) & =\frac{1}{3}[4 g(0)-1],
\end{aligned}
$$

Note that if the negative sign of the local-field factor at high $\omega$, by Eq. (36), persists at finite $\omega$ then we have the possibility that a pole in $\chi_{S}(\mathbf{q}, \omega)$ exists ${ }^{13}$ for real $\omega$ and sufficiently large $r_{s}$. Physically, this collective mode corresponds to long-wavelength spin-wave modes, undamped and oscillating at frequencies given by the solution to

$$
1+V(\mathbf{q}) G_{-}(\mathbf{q}, \omega) \bar{\chi}_{0}(\mathbf{q}, \omega)=0
$$

In two dimensions the limiting forms of the highfrequency local-field factors are

$$
\begin{aligned}
G_{-}^{(\infty)}(\mathbf{q} \rightarrow 0) & =\frac{1}{4 \pi n q} \int_{0}^{\infty} d k k^{2}[\tilde{S}(k)-S(k)], \\
G_{-}^{(\infty)}(\mathbf{q} \rightarrow \infty) & =g(0) .
\end{aligned}
$$

\section{DISCUSSION}

The physical concept behind the introduction of the local-field factor is identical in spirit to the methodology of DFT in which xc effects are incorporated in an effective local field experienced by each electron. In fact, within the frequency-dependent linear response formalism ${ }^{14}$, the unpolarized spin-antisymmetric local-field factor is simply related to the xc energy functional $E_{\mathrm{xc}}[n]$ via,

$$
G_{-}(\mathbf{q}, \omega)=\frac{K_{\mathrm{xc}}^{\uparrow \downarrow}(\mathbf{q}, \omega)-K_{\mathrm{xc}}^{\uparrow \uparrow}(\mathbf{q}, \omega)}{2 V(\mathbf{q})} \equiv-\frac{K_{\mathrm{xc}}^{-}(\mathbf{q}, \omega)}{2 V(\mathbf{q})}
$$

where $K_{\mathrm{xc}}^{\sigma \sigma^{\prime}}(\mathbf{q}, \omega)$ is the Fourier-transform of the xc kernel of TDSDFT,

$$
K_{\mathrm{xc}}^{\sigma \sigma^{\prime}}\left(\mathbf{r}-\mathbf{r}^{\prime}, t-t^{\prime}\right) \equiv \frac{\delta^{2} E_{\mathrm{xc}}[n]}{\delta n_{\sigma}(\mathbf{r}, t) \delta n_{\sigma^{\prime}}\left(\mathbf{r}, t^{\prime}\right)}
$$

From Eq. (36) and Eq. (39) we now note that $\lim _{\mathbf{q} \rightarrow 0} \lim _{\omega \rightarrow \infty} K_{\mathrm{xc}}^{-}(\mathbf{q}, \omega)$ diverges as $q^{-2}$ in both two and three dimensions in notable contradistinction to $K_{\mathrm{xc}}^{+}(\mathbf{q}, \omega)$ which is well-behaved in the same limit. This striking behavior of the spin-antisymmetric exchangecorrelation kernel does not by itself lead to any concern since the corresponding macroscopic quantities (i.e. $\chi_{S}$ as given by Eq. (30) remain well-defined in the low- $q$ and high- $\omega$ limit and it follows that the observable (i.e. induced magnetization) also remains well-defined. Physically, the divergence in momentum-space implies that $K_{\mathrm{xc}}^{-}(r, \omega)$, and hence the xc hole, is of infinite extent, i.e. it decays very slowly, especially so in two dimensions. The divergence is then a signature of a transition from a bounded xc kernel to an unbounded one. Thus a strict local-density approximation $(\mathrm{LDA})^{14}$ for the $\mathrm{xc}$ potential becomes untenable in time-dependent systems, at least in the high-frequency regime. In other words, the dynamical local field cannot be specified by the local density alone and other approaches to TDSDFT are required. This divergence is particularly potent given that it occurs even in a homogeneous system, as emphasised by Vignale ${ }^{15}$ who very recently reported similar conclusions about the dynamic spin response at finite- $\omega$.

One possible remedy is via the weighted density approximation $^{16}$ which retains nonlocality and has been demonstrated to give more accurate results than functional approximations involving local-density contributions. Indeed it has been argued ${ }^{17.18}$ that such an approach should be able to recover at least part of the the full Van der Waals interaction, the origin of which is clearly due to dynamical correlation between electrons, and where it is well known that the conventional LDA fails. Another approach which overcomes the limitations of the LDA but nonetheless still permits a local description is current density functional theory ${ }^{19.20}$ where the local current density is required in addition to the local density to describe time-dependent inhomogeneous systems.

Finally, we note that many-body calculations based on Hedin's $G W$ approximation 21 take correlations into account directly without invoking the local mean-field approximations of DFT, and have recently ${ }^{22}$ been demonstrated to yield accurate ground-state energies of inhomogeneous systems where LDA fails drastically. However, such many-body approaches are numerically intensive and it remains to be seen whether they can be successfully applied to excited states within feasible computational effort. 


\section{Acknowledgments}

This work was supported by the NSF under Grant No. DMR-9988576.

1 N. Iwamoto, Phys. Rev. A 30, 3289 (1984).

2 B. Davoudi, M. Polini, G. F. Giuliani, and M. P. Tosi, Phys. Rev. B 64, 233110 (2001).

3 B. Davoudi, M. Polini, G. F. Giuliani, and M. P. Tosi, Phys. Rev. B 64, 153101 (2001).

4 M. Corradini, R. D. Sole, G. Onida, and M. Palunmo, Phys. Rev. B 57, 14569 (1998).

${ }^{5}$ R. D. Puff, Phys. Rev. 137, 406 (1965).

${ }^{6}$ G. S. Atwal, Ph.D. thesis, Cornell University (2002).

7 H. B. Singh and K. N. Pathak, Phys. Rev. B 10, 2764 (1974).

8 B. Goodman and A. Sjolander, Phys. Rev. B 8, 200 (1973).

9 I. D'Amico and G. Vignale, Phys. Rev. B 62, 4853 (2000).

10 C. F. Richardson and N. W. Ashcroft, Phys. Rev. B 50, 8170 (1994).

11 X. Zhu and A. W. Overhauser, Phys. Rev. B 30, 3158 (1984).

12 G. Niklasson, Phys. Rev. B 10, 3052 (1974).
13 K. Utsumi and S. Ichimaru, Phys. Rev. B 28, 1792 (1983).

14 E. K. U. Gross and W. Kohn, Phys. Rev. Lett. 55, 2850 (1985).

15 G. Vignale, Bull. Am. Phys. Soc. 99, 99 (2002).

16 O. Gunnarsson, M. Jonson, and B. I. Lundqvist, Phys. Rev. B 20, 3136 (1978).

17 A. C. Maggs and N. W. Ashcroft, Phys. Rev. Lett. 59, 113 (1987).

18 K. Rapcewicz and N. W. Ashcroft, Phys. Rev. B 44, 4032 (1991).

19 G. Vignale and W. Kohn, Phys. Rev. Lett. 77, 2037 (1996).

20 G. Vignale, C. A. Ullrich, and S. Conti, Phys. Rev. Lett. 79, 4878 (1997).

21 L. Hedin, Phys. Rev. 139, A796 (1965).

${ }^{22}$ P. Garcia-Gonzalez and R. W. Godby, Phys. Rev. Lett. 88, 056406 (2002). 